chrysophanic acid ointment (thirty grains to the ounce is usually strong enough), and I have found it a very effective remedy, but there are great drawbacks to its general use. First, it stains everything with which it comes in contact, and in the second place we are uncertain as to the amount of inflammation it may set up ; some children bear it well, while in others it produces so much irritation, swelling, and discolouration of the skin as to alarm those who use it. It must therefore be used with caution, and patients should be warned of its properties; nevertheless, I repeat, it is a very effective remedy.

Your success in the treatment of ringworm will depend on your choosing your remedies with judgment, being guided in your choice by the circumstances of the case, and always bearing in mind that you have to steer, as it were, between setting up too much inflammation on the one hand and not using sufficiently strong means on the other. Whatever treatment, however, you adopt, you will meet with a certain number of cases that defy your best efforts, and that get well only perhaps after years of tedious care. As a rule, shaving the head is quite unnecessary, but the hair should be kept quite short. Skull-caps are best avoided, as liable to propagate the disease. With regard to epilation, which is so largely used in France as a mode of treatment, I do not find that it is often necessary ; it is, however, occasion. ally useful. Take, for example, the case of a boy anxious to return to school, who has a patch of chronic tinea tonsurans. In this case the extraction of the diseased hairs will shorten the treatment required, and enable him to return to school cured somewhat sooner than would otherwise be possible. Lastly, most observers agree that ringworm is often associated with a generally unhealthy condition of the skin, which is badly nourished. Under these circumstances, tonics such as iron and arsenic are often useful. This is quite in accordance with the fact that many strictly local affections are influenced by general treatment.

\section{A NOTE ON}

\section{THE ROUTINE USE OF THE HYPODERMIC SYRINGE AS AN AID TO DIAGNOSIS.}

\author{
By W. S. GREENFIELD, M.D., F.R.C.P.,
} ASSISTANT-PHYSICIAN TO ST. THOMAS'S HOSPITAL.

THE employment of some instrument as an aid to the diagnosis of the nature of fluid effusions, and their distinction from solid tumours has long been a habit in surgery. The old grooved needle has now given way to more delicate hollow needles. Since the introduction of the aspirator, physicians have been led to look with far less dread upon the operation of puncturing internal organs, and those who have been most bold in its use have had least occasion to fear any evil result. I have no doubt that there are many who, like myselt, are in the habit of employing the common hypodermic syringe as an everyday aid to diagnosis and guide to treatment, and they may consider any advocacy of its use a work of supererogation; but my own experience has convinced me that a very large number of physicians do not avail themselves of it, and that those who do, resort to it but rarely. For some years I have constantly and systematically employed it, more especially in the diagnosis of chest diseases ; and the occasions for its aid are so frequent in my own experience that similar instances can hardly fail to occur in the practice of all. And I must say that I have never seen any ill result follow its use, whilst it has furnished invaluable information in a large number of cases.

Let me speak first of chest diseases. It is especially in cases of pleural effusion that it comes into requisition. We may have signs pointing to, but not decisively indicating, the presence of fluid, or may be in doubt whether they in. dicate consolidation or fluid accumulation; or, on the other hand, we may be sure there is fluid, but uncertain whether it is serous, purulent, or sanguineous. Our doubts are immediately settled by a puncture. Again, when fluid has been discovered, we may be uncertain whether there is only one effusion, which we may relieve by aspiration, or whether there are other loculated effusions which would not thus be reached. By two or three punctures in different situations we may be able to decide this point, finding that the fluid has different characters at different positions. To give a case in point :-A patient who had long suffered from symptoms of mediastinal tumour of doubtful nature, with consolidation of the whole of one lung, but with little expectoration, suddenly began to bring up large quantities of fetid pus. The question whether this was due to an unrelieved empyema, or to purulent accumulation pent up in the lung itself and suddenly finding an exit, seemed an important one for prognosis and treatment. A puncture near the lower part of the lung withdrew perfectly clear serum ; another, a little higher up, removed some fetid pus, in every respect like that expectorated, together with some air. Immediately afterwards a small quantity of bright-red blood was coughed up. These facts, taken together, were indicative that there was a loculated pleurisy, and that the second puncture had entered the lung itself, and removed from eroded lung-tissue some fetid pus, as was proved a week or two later by the autopsy. I may add that this is the only instance I can recall of an apparent ill result from such puncture, and here no real harm was done.

In very many cases both diagnosis and treatment must be determined by the nature of the fluid accumulation. Let me especially instance the very obscure and difficult class of cases in which, with but little evidence of acute illness, we find dulness with loss of respiratory sounds, and slight or no friction at the lower part of the right lung. We may and ought to be largely guided in our diagnosis by the history of the case and by the physical signs, yet how often is the conclusion erroneous if we rely upon these alone. Who has not seen cases of hydatid or abscess of the liver diagnosed as simple pleurisy? And how often are these cases complicated, either as the result of a secondary or coincident pleural inflammation, or as a sequel of perforation. Whatever be our views as to the desirability of evacuation by puncture or otherwise, there can be hardly two opinions as to the wisdom of ascertaining the actual state of affairs, that our general treatment and prognosis may be guided by it. Let me give an example from a recent case, which is one of pretty com. mon occurrence. A gentleman, twenty-six years of age, of strongly phthisical family, and with a markedly phthisical constitution, has suffered for two months from severe cough with muco-purulent expectoration, which of late has become puriform and often blood-stained. Rather less than five months ago he had acute dysentery, followed during convalescence by right pleurisy. Physical examination shows dulness with loss of respiratory and rocal sounds in the lower third of the right axilla, and to a less marked degree at the back; very little else abnormal in the chest; no enlargement or tendermess of the liver; no history of hepatic symptoms. There is withal just that amount of flattening, prolonged expiration, \&c., at both apices, which would make one suspicious of phthisis in a patient of his antecedents and history, and this suspicion is strongly confirmed by other symptoms. But for the history of dysentery, one could hardly have doubted that it was a case, such as one often sees, of latent pleurisy in a phthisical subject. But the hypodermic syringe withdraws thick, curdy pus, mixed with blood, and on comparison with the sputum the characters of the two are found to be identical. The character of the fluid together with the history leave but little doubt that the case is one of hepatic abscess which, after perforation into the pleura, is making its way through the lung. The circumstances of the case were such as to render it very important that the diagnosis should be made at once, whilst the general symptoms indicated the desirableness of speedy treatment. The course of the case subsequent to aspiration of the fluid has been strongly confirmatory of the advantages of the method pursued.

It would be easy to multiply cases in which the use of the hypodermic needle has given valuable information as to the presence or nature of pleural effusions. Further, it may be employed both as an aid to treatment or for actual treatment of such cases. In many cases in which it is decided to aspirate, or to introduce a trocar, it is very desirable to determine precisely the lowest point at which fluid readily flows, and, in the case of loculated effusions, to fix exactly the site of puncture. By no means can this be done so readily and so exactly as with the hypodermic needle. I am in the habit in all such cases of using this first, and often make three or four punctures to decide upon the most favourable spot. 'This having been done, we can decide exactly what sized needle or trocar to use, and how deeply it must go. 
In the case of small effusions, and also in empyema in infants, we may use the hypodermic syringe alone, repeatedly removing small quantities of fluid.

In many other circumstances we may also employ this method. In the case of abscesses seated near the surface of the body, such as some hepatic abscesses, perityphilitic abscesses, and the like; and in a large number of swellings in limbs, \&c., of doubtful nature, which come rather under the care of the surgeon, we may gain most valuable information. But this is not a fitting occasion for pointing out all the uses to which it may be applied.

Before summing up the points in favour of its use, let me say a word of the kind of instrument to be used, and the method of use. First of all, to fulfil all the conditions, it is essential that the needles should be fine, with a grooved and very sharp point, that they should be made of polished steel, and that they should be kept well tempered and scrupulously clean. The syringe should be rather large, made of glass, with metal fittings, and the piston always well soaked. The junction of the needle with the syringe must be thoroughly air-tight. It is essential that the operation of puncture should be as nearly as possible painless, that we may be able to tell the patient that it is less than a pin prick, and justify our statement. The needles should not be less than one and a quarter, nor as a rule more than one and three-quarter inches in length. The diameter should not, I think, exceed one millimetre. These details are not unimportant, for a very large number of common hypodermic syringes do not fulfil these indications, and I believe that they are essential if the pain is to be reduced to a minimum. Where it is desirable only to remove a moderate quantity of fluid, from one to one-and-a-half ounce (even a smaller quantity may sometimes be removed in empyema of infants with advantage), I employ a larger syringe, holding about an ounce, which is screwed on to the needle in situ after screwing off the small syringe.

As to the method of use, little need be said. The site of puncture having been determined upon, the ball of the left forefinger is firmly pressed into the intercostal space at the spot indicated, and the needle plunged boldly in close to the point of the nail. The pressure of the finger seems to deaden the sensibility of the skin, and the finger serves as a guide to prevent puncture of the rib. By way of preface to the patient, it is rarely necessary to say more than "Do you mind a prick?" and almost before the answer " $\mathrm{Oh}$, no" is completed, the whole operation is over. Often I have been asked when I was going to begin, when I had already finished. The needle should be withdrawn rapidly, the finger being pressed against the skin close to it. Any long preface or display of instruments is to be avoided; for even a moment's apprehension may be prejudicial to a nervous patient; and the small size of the instrument is in this respect a great advantage.

I have no doubt that $I$ shall be accused by some of a prolix narration of trivialities, or of magnifying the importance of indifferent details; whilst there will be some who consider other means of diagnosis always sufficient, and regard the routine exploration by the hypodermic syringe as a needless barbarity, to be employed only by those who belong to what a facetious friend of mine calls the "toasting-fork school."

To the first I must answer that nothing is unimportant of explanation which is frequently useful and often neglected or misunderstood. It has taken some years to convince me that so obvious and simple an aid to diagnosis needed any elucidation or enforcement ; it is only because I find that it is in practice often ignored that I venture to urge its use, and to point out how it may best be applied.

But to those who object that it is needless or dangerous another answer must be given. There are some who rely upon external signs to the exclusion of other more exact methods, and who consider it a lowering of the standard of medical diagnosis to resort to such means as puncture. They prefer to rely exclusively upon the signs given by auscultation and percussion, and the position and shape of dulness, \&c., as furnishing greater scope for the exercise of diagnostic acumen. On similar grounds many London physicians laughed at the stethoscope and auscultation when Laennec's methods were first used in England by Hope and others. I would be amongst the last to advocate the careless use of the hypodermic needle; in every case all other modes of physical examination ought to be first resorted to. But since diagnosis can be completed by the one, and only pushed to a certain degree by the other means, there can be no reason why both should not be employed in doubtful cases.

The possible dangers $I$ have never known to occur. The shortness and small size of the needle, and the small suctionforce employed, are a guarantee against some of the evils which may arise from the longer needles and greater force of the aspirator. With ordinary cleanliness there is no possibility of giving rise to septic infection, nor can air enter the pleura, or suppuration be set up. I do not deny that puncture of the heart or of the intestines may be serious, and some would prefer not to puncture the peritoneum, though I have repeatedly done so without any ill effects, as have many others. But only by unjustifiable ignorance or carelessness could either heart or intestine be accidentally punctured. The latter may, as we know from treatment of intestinal obstruction, be sometimes punctured without harm, though not without risk, but with the heart there is always danger. But $I$ do not think that any harm could result from the puncture of an aneurismal sac, or of an intercostal artery or vein, with so fine a needle as I have indicated.

Palace-road, S.E.

\section{SOME PRACTICAI REMARKS ON THE USE OF MARTIN'S BANDAGE.}

By WILliaM S. BYRNE, A.B., M.B., M.Ch. T.C.D. SENIOR HOUSE-SURGEON, METROPOLITAN FREE HOSPITAL.

WHEN Dr. Martin, some time ago, introduced to the notice of the medical profession his india-rubber bandage, and spoke so highly of its curative effects in certain forms of surgical disease, it was decided that the Metropolitan Free Hospital should obtain a supply for the use of the patients, and in suitable cases the satisfactory results obtained amply prove the accuracy of Dr. Martin's remarks in his valuable pamphlet. Amongst the cases treated by his bandage were chronic ulcers and varicose veins of the leg, acute and chronic synovitis of the knee-joint, and chronic bursitis of the elbow-joint, and to these cases $I$ wish to draw attention. In the case of ulcers and varicose veins the bandage was applied in the morning before rising, and taken off at night when in bed. Generally when the bandage was taken off a number of ridges of various depth were seen in the skin, the result of the upper edge of each fold of the bandage pressing upon the leg more tightly than the lower edge, but this did not appear to cause any injury, and in no case did any abrasion or excoriation of the skin occur. In a few of the cases the toes and part of the foot below the bandage became slightly swollen, but the patients never complained of pain, and as a rule expressed themselves as feeling most comfortable.

The great utility of this treatment in ulcers of the leg may be shown by the fact that in the cases quoted below the patients were unable to leave their work to lie in bed for a couple of months, and had been attending hospital, one for a space of nine months, and another for five months, without deriving any considerable benefit, but from the time of application of Martin's bandage they improved, and were cured in five weeks time.

With regard to the varicose veins, hardly enough time has elapsed to allow me to judge of the efficacy of the treatment, but in one case, where the vein had nearly burst at the ankle, the bandage seemed, by the pressure exercised, to exert a beneficial influence; for the spot had healed in a week, leaving but little soreness, and the patient seemed to be doing well. It appears to have an advantage over the elastic stocking in those cases-viz., that it may be applied as tightly or as loosely as may be deemed requisite, and that it does not lose its elasticity.

In the case of acute syuovitis of the knee-joint it exercised a wonderful influence in effecting a cure in nine days, and I have no doubt would have acted just as beneficially in the subacute case of the same disease had the patient been allowed to remain under treatment. In one case of chronic bursitis of the elbow-joint the bandage did not do any good, but this I apprehend was owing to the number of milletseed bodies found in the tumour keeping up the irritation, 